\title{
Dental Occlusion: Proposal for a Classification to Guide Occlusal Analysis and Optimize Research Protocols
}

\author{
Casazza Estelle ${ }^{1}$, Ré Jean-Philippe ${ }^{2}$, Giraudeau Anne ${ }^{3}$, Parfu Anne ${ }^{4}$, Orthlieb Jean-Daniel ${ }^{5}$
}

\begin{abstract}
Aim: Dental occlusion often rhymes with confusion, discrediting many research protocols. The profession seems to be in "major chaos about occlusion." This may be due to the lack of a precise classification of occlusal dysfunctions. We suggest using a classification based on the separation of three occlusal functions, with the identification of precise, objective clinical diagnostic criteria. This article aims to define a precise classification of occlusal functions, in order to be able to establish a positive diagnosis of occlusal disorders. This occlusal analysis method could then be used in the daily practice of dentists and orthodontists, with a view to align epidemiological studies that focus on occlusion, in order to obtain results capable of comparison in different studies.
\end{abstract}

Materials and methods: A analysis of the literature in PubMed database published between the early 1970s and the present day identified many confusing definitions of occlusal disorders. In this paper, we propose the separation of occlusal functions into three subcategories: Stabilizing, centering, and guiding functions, defining three different subcategories of occlusal disorder.

Results: Occlusal function allows the definition of three kinds of malocclusion: Stabilizing dysfunction, centering dysfunction, and guiding dysfunction. The individualization of clear subcategories could allow the study of the more pertinent impacts of pathogenic malocclusion.

Conclusion: This classification of occlusal functions or dysfunctions allows lines to be drawn between different occlusal situations that are frequently confused, such as a loss of posterior occlusal support and loss of occlusal vertical dimension, infra-occlusion and loss of posterior support, short or reduced dental arch, reversed and scissor occlusion, sagittally and transversely deflected mandible, posterior occlusal interference and balancing contact, as well as natural and iatrogenic malocclusion.

Clinical significance: An occlusal analysis that makes use of the three occlusal functions, "stabilizing, centering, guiding," could offer diagnostic standardization. It may also allow the avoidance of incorrect interpretations. Therefore, this occlusal function classification may be relevant to many fields, for instance, for epidemiological studies of occlusion and the periodontium, TMD and occlusion, or TMD and orthodontic treatment. Keywords: Dysfunctions, Malocclusion, Occlusal functions, Occlusion.

The Journal of Contemporary Dental Practice (2021): 10.5005/jp-journals-10024-3113

\section{INTRODUCTION}

The relationship between temporomandibular disorders (TMD) and dental malocclusion is still a controversial topic, although as noted by Manfredini, "Dental occlusion is the core of dentistry." 1 Türp observed: "Decades of research have progressively shed light on many issues relating to the management of occlusion in clinical practice," but many gray areas persist. ${ }^{2}$ It is necessary to reframe dental occlusion within a wider context: Skeletal typology, parafunctions, biological field, psychosocial dimension, hypervigilance, etc., but also, we have to think about more pertinent definitions of different occlusal disorders. Excluding the TMD field, the various schools of thought on the subjects of occlusal diagnosis and the reconstruction of dental occlusion offer opposing views with regard to theoretical concepts, diagnostic tools, and therapeutic orientations. ${ }^{3}$ The profession seems to be in "major chaos about occlusion." ${ }^{4}$

In 2017, the glossary of prosthodontic terms defined dental occlusion as "the static relationship between the incising or masticating surfaces of the maxillary or mandibular teeth." ${ }^{5}$

It seems paradoxical to define a static relationship by referring to functions that are moving actions. The definition of the simple term "dental occlusion" seems confusing. We could propose the following definition: Opposing situations corresponding to all the possible states of reciprocal confrontations of two dental arches. This, there are numerous mandibular occlusion positions.
${ }^{1-5}$ Aix-Marseille University, School of Dentistry, Marseille, France; APHM, Timone Hospital, Marseille, France

Corresponding Author: Casazza Estelle, Aix-Marseille University, School of Dentistry, Marseille, France; APHM, Timone Hospital, Marseille, France, Phone: +33 678371907, e-mail: estelle.casazza@ univ-amu.fr

How to cite this article: Estelle C, Jean-Philippe R, Anne G, et al. Dental Occlusion: Proposal for a Classification to Guide Occlusal Analysis and Optimize Research Protocols. J Contemp Dent Pract 2021;22(7):840-849.

Source of support: Nil

Conflict of interest: None

Malocclusion is defined by: " 1 . any deviation from a physiologically acceptable contact between the opposing dental arches; 2 . any deviation from a normal occlusion. ${ }^{15}$

What is normal? Does normal mean ideal, or the most frequently observed situation?

It would seem important to first clarify the term, "malocclusion," with these different subcategories. Indeed, "malocclusion" is very difficult to define, in particular its possible pathogenic repercussions. Normocclusion and malocclusion are not black and white; they represent a continuum of gray levels. Because terminology and classification are at the root of science, we propose to identify

(c) Jaypee Brothers Medical Publishers. 2021 Open Access This article is distributed under the terms of the Creative Commons Attribution 4.0 International License (https://creativecommons.org/licenses/by-nc/4.0/), which permits unrestricted use, distribution, and non-commercial reproduction in any medium, provided you give appropriate credit to the original author(s) and the source, provide a link to the Creative Commons license, and indicate if changes were made. The Creative Commons Public Domain Dedication waiver (http://creativecommons.org/publicdomain/zero/1.0/) applies to the data made available in this article, unless otherwise stated. 
four occlusal situations within this continuum: Normocclusion, functional occlusion, functional malocclusion, and pathocclusion.

- Normocclusion: The ideal concept of the occlusal relationship described in textbooks, rarely completely observed in nature.

- Functional occlusion: The presence of some characteristics of normocclusion with individual variants; the target of therapeutic intervention when dental treatment is indicated due to dental status.

- Functional malocclusion: Occlusal dysfunctions without obvious structural impairments or functional impotency. This adaptive function (called eufunction) is a common situation in the natural development of the individual dentition.

- Pathocclusion: Obvious occlusal disorders characterized by clear dental instability and functional impotency; this occlusal pathofunction is corrected in routine dentistry.

From a mechanical perspective, it is probably easy to obtain a consensus on the criteria for the two extreme conditions, normocclusion and pathocclusion. It is common sense to correct "pathocclusion" and to recommend no intervention in cases of "functional occlusion." The problem lies in differentiating functional occlusion from functional malocclusion because the boundary is difficult to define; "functional malocclusion" represents a gray area for therapeutic decisions: Should occlusion be corrected or not? Answering this question requires the identification of specific, positive, clinical signs for each occlusal disorder. This is the goal of this classification proposal.

Evidence-based medicine entails the systematic, explicit, and judicious implementation of the best evidence in patient care. The most reliable sources of evidence are high-quality systematic reviews and randomized controlled trials. ${ }^{6}$ This methodological process must firstly be based on an appropriate initial group composition particularly from the perspective of occlusal disorders. So, occlusal disorders need to be clearly defined before studying their possible negative impact on teeth, the periodontium, the temporomandibular joint, or muscles. Better definitions and a consensus are needed to define which malocclusion variants could be potentially pathogenic.7 An explanation for the ongoing controversy regarding "occlusion" and "temporomandibular disorders" could be definition-based. ${ }^{8}$ For example, TMDs have an appropriate classification system, detailing inclusion criteria for the different subcategories; this is not the case with occlusal disorders. The purpose of this article is to try to clarify occlusal categories using a classification based on the separation of three occlusal functions, with specific, positive clinical diagnostic criteria, in order to align the research of epidemiological studies that focus on the role of occlusion, so as to obtain results in different studies that are capable of being compared.

\section{Materials and Methods}

From the search in PubMed database with the following keywords: "dental occlusion," "malocclusion," "interference," "prematurity," "occlusal disorders," and "occlusal dysfunction" completed by manual searches in various textbooks, an analysis of the literature published between the early 1970s and the present day was conducted to note the criteria for describing the various anomalies of the dental occlusion. This analysis identified many confused definitions of occlusal disorders. In a nonexhaustive list, we can note confusions between:

- Loss of posterior occlusal support and loss of occlusal vertical dimension
- Infra-occlusion and loss of posterior support

- Overocclusion and occlusal interference

- Posterior occlusal interference and balancing contact

- Short dental arch or reduced dental arch

- Sagittal and transversal deflective occlusal contact

- Reversed occlusion and scissors occlusion

- Functional guidance with overguidance

To reduce the confounding factors, observed in many articles, in the description of the occlusion, from this analysis of the literature, we propose an occlusal classification.

We propose separating "occlusal functions" into three subcategories as follows: Stabilizing, centering, and guiding (Figs $1 \mathrm{~A}$ and $1 \mathrm{~B})$.

- Stabilizing function (stability): What is the quality of the Intercuspal position (ICP)?

- Centering function (spatial position): Where is the ICP? Are the condylar positions imposed by the ICP orthopedically stable for the TMJs?

- Guiding function (dynamic movement): How to attain the ICP? The occlusal control of mandibular movements to access the ICP.

Then, the triad of occlusal functions (stabilizing, centering, and guiding) will allow the definition of three different subcategories of occlusal dysfunctions that are often confusing for many practitioners, and also in numerous research studies:

- Stabilizing dysfunctions: An unstable ICP; this involves a static analysis.

- Decentering dysfunctions: An ICP deflected from the reference centric position (RCP); this involves a static comparison of two mandibular reference positions (ICP-RCP).

- Guiding dysfunctions: Occlusal interferences that cause deviations or limitation of mandibular movement when returning to the ICP. This involves a kinematic analysis.

\section{Results: Criteria for the Analysis of OcClusion and Malocclusions}

The results around the analysis of confounding factors led to classifying them according to the triad of occlusal functions in order to distinguish those relating to stability from those which influencing the condylar position in maximal intercuspal position, and from those which influencing mandibular movements.

\section{Stabilizing Function or Dysfunctions}

Stability is crucial to understand the role of occlusion.

In the maximal intercuspal position or ICP, the stabilizing function ensures stable mandibular support to facilitate swallowing and clenching, the two operations performed most frequently by the masticatory apparatus. This immediate, full interlocking of the dental arches establishes a primary mandibular stabilization, inducing neuromuscular facilitation and decreasing the need for muscle recruitment ${ }^{9-11}$ :

- The homogeneous, brief, symmetrical, synergistic, and, therefore, ergonomic recruitment of muscles,

- A mandible that is more frequently in the rest position (a stable and precise ICP creates neuromuscular "confidence," as opposed to recurrent overactivity),

- Easier postural balance. 


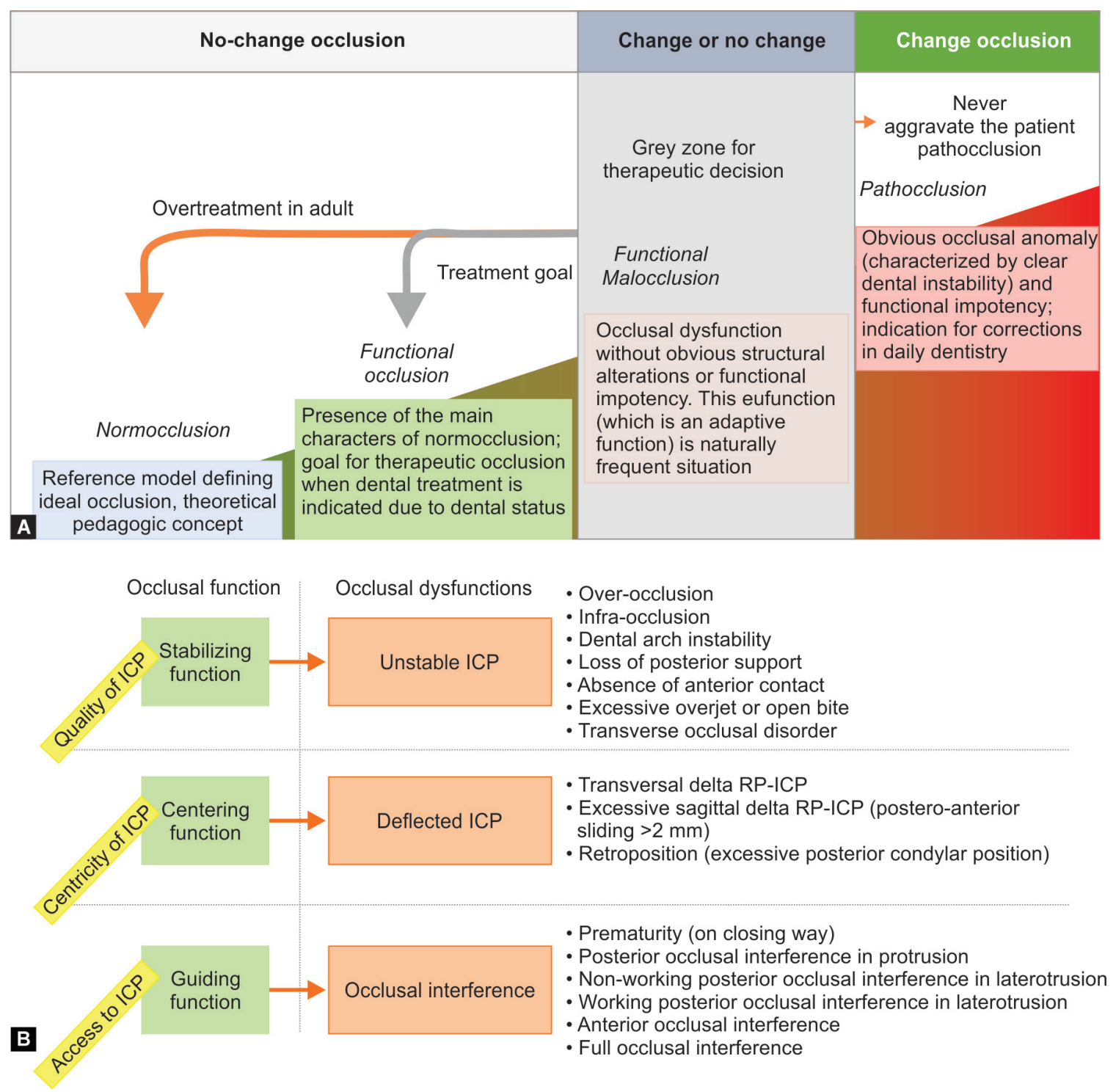

Figs 1A and B: Occlusion categories and classification of occlusal dysfunctions, with: (A) Occlusal categories from a functional perspective; (B) Triad classification of occlusal functions or dysfunctions

\section{What does Occlusal Stability Mean?}

The term "occlusal stability" is defined in prosthodontics as the equalization of tooth contacts that prevents tooth movement after closure. ${ }^{5}$ ICP quality is one of the factors that ensures the absence of tooth migration. The stabilizing function, therefore, entails intra-arch stability (stability of the teeth) and interarch stability (mandibular stability). These stabilities are achieved by the distribution and precision of the multiple occlusal contacts, consolidated over the entire arch by the proximal contacts. ${ }^{12}$

Stability in biology is always relative; so, the ICP changes constantly during the dentition periods (deciduous and adult). During aging, the stability of the dental arches is globally maintained, although there is always natural, low level, but continuous migration due to dental abrasion, occlusal wear (offset by the natural supra-eruption of teeth), and proximal wear (compensated by the natural mesial drift of the teeth). The development of slight mandibular anterior crowding could be often the natural expression of these phenomena. ${ }^{13}$

\section{Criteria of Occlusal Stability}

Basically, the stability of each dental organ in its arch determines the absence of tooth migration (a sign of instability). Elementary mechanical principles suggest that this stability is related to:

- The correct axial inclination of the tooth,

- Dental arch continuity ensured by interproximal contacts (no diastema) (Figs 2A and 2B),

- The distribution of punctiform contacts over the entire arch,

- Sagittal stability: Occlusal contacts on opposite sides of the tooth-mesial or distal (one tooth/two teeth),

- Transverse stability: Occlusal contacts on opposite sides of the tooth-bucco or lingual. These opposite contacts are more necessary in the transverse direction than the sagittal 


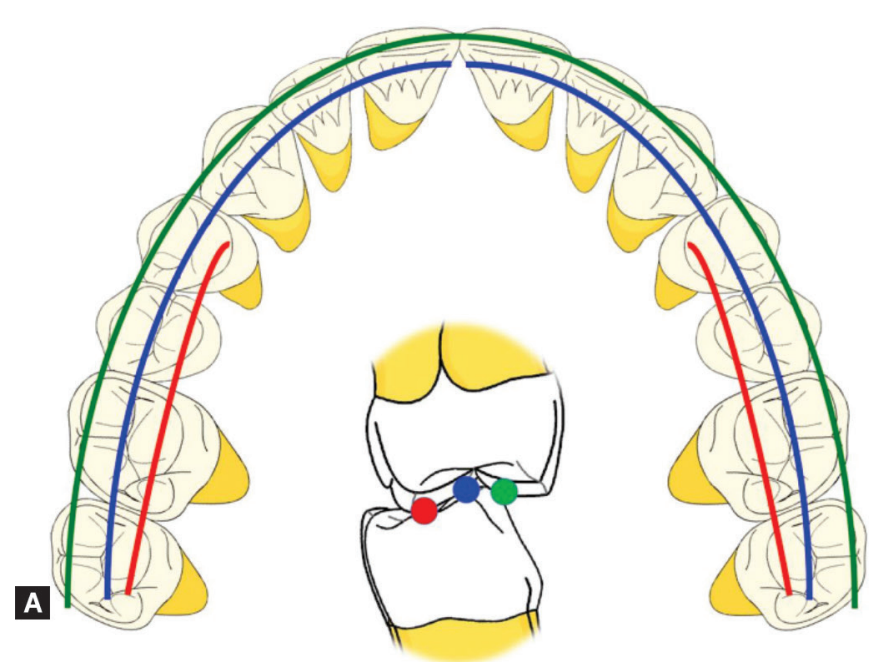

- Esthetic functional arch (guiding) Centric arch (sagittal stability) - Lingual active arch (transversal stability

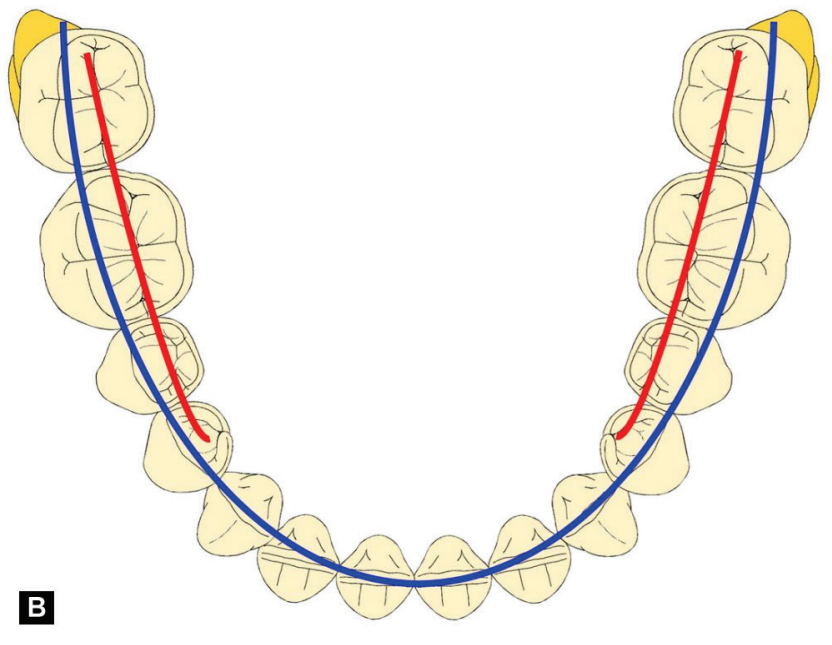

- Lingual active arch - Centric arch (sagittal stability)

Figs $2 \mathrm{~A}$ and $\mathrm{B}$ : The three occlusal arches. The stabilizing arch (blue line) is the only occlusal arch, which is both continuous and involves both dental arches; it is only at this level that the occlusal plane can be defined. (A) Maxillary view; (B) Mandibular view

direction as the latter is offset by the continuity of the dental arch (proximal stability).

\section{Dysfunctions of Occlusal Stability}

Within the stabilizing function category, different types of dysfunctions of occlusal stability must be distinguished (Fig. 3):

- Globally unstable ICP: Instability of the dental arch (dental migration, rotation, and supra-eruption) (Fig. 3A).

- Overocclusion: Excessive occlusal contact (which is always iatrogenic), concentrated on one or more units (often prosthetic) preventing complete initial ICP.

- Infraocclusion: One or more dental elements are present but do not participate in the ICP; they are in under contact (Fig. 3B), and sometimes this is very slight.

- Loss of posterior occlusal support by exocclusion, missing, or broken tooth (Fig. 3C).

- Anterior occlusal instability: Absence of anterior occlusal contact in ICP, excessive overjet or anterior open bite (Fig. 3D).

These types of the occlusal disorders may generate dental overload, dental migration, joint overload, or difficulties with muscle recruitment.

The "posterior crossbite" is a perfect example to underline the fact that the stabilizing function concept allows a better understanding of occlusal disorders. There is some confusion in the literature concerning the posterior crossbite concept. Posterior crossbite is usually defined as "one or more teeth of the posterior group (from canine to second molar) in an irregular (at least one cusp wide) buccolingual relationship, with one or more opposing teeth. ${ }^{14}$ It seems necessary to rename the different types of transverse occlusal disorders. The impact on occlusal stability is clearly different depending on whether the transverse disorder involves half a cusp (end-to-end), one cusp (reversed occlusion), or two cusps (exocclusion) (Fig. 4). The classic term "crossbite" places very different occlusal situations in the same box. So, this term cannot be used in the study of pathogenic incidences of transverse occlusal disorders.

- Transverse normocclusion: Occlusal stability is optimal (the largest cusp in the largest fossa).

- End-to-end occlusion: Lack of occlusal stability (mandibular instability)

- Reversed occlusion: Stabilizing function is maintained (the occlusal relationship is stable; occlusal interferences occur frequently)

- Scissor occlusion: Occlusal stability is absent (strong working interferences)

- Reverse scissor occlusion: Occlusal stability is absent (strong nonworking interferences)

$$
\begin{aligned}
& \text { Dysfunctions of occlusal stability } \\
& \text { - Globally unstable ICP } \\
& \text { - Overocclusion } \\
& \text { - Infraocclusion } \\
& \text { - Loss of posterior occlusal support } \\
& \text { - Excessior occlusal instability } \\
& \text { - Transverse occlusal disorders }
\end{aligned}
$$

\section{Centering Functions or Dysfunctions}

\section{Centric Relation}

According to Okeson, centric relation (CR) designates an optimal orthopedically stable joint position obtained when the condyles are in their most anterosuperior position in the articular fossa. ${ }^{15}$ TMJ structures are often seen only from the lateral view, but undoubtedly, the dento-musculoskeletal structures of the masticatory apparatus are globally symmetrical. Logically, the ICP must provide a symmetrical mandibular position with respect to the craniofacial unit. This means that under loading conditions (e.g., clenching in ICP), the TMJ must be able to absorb the load, 

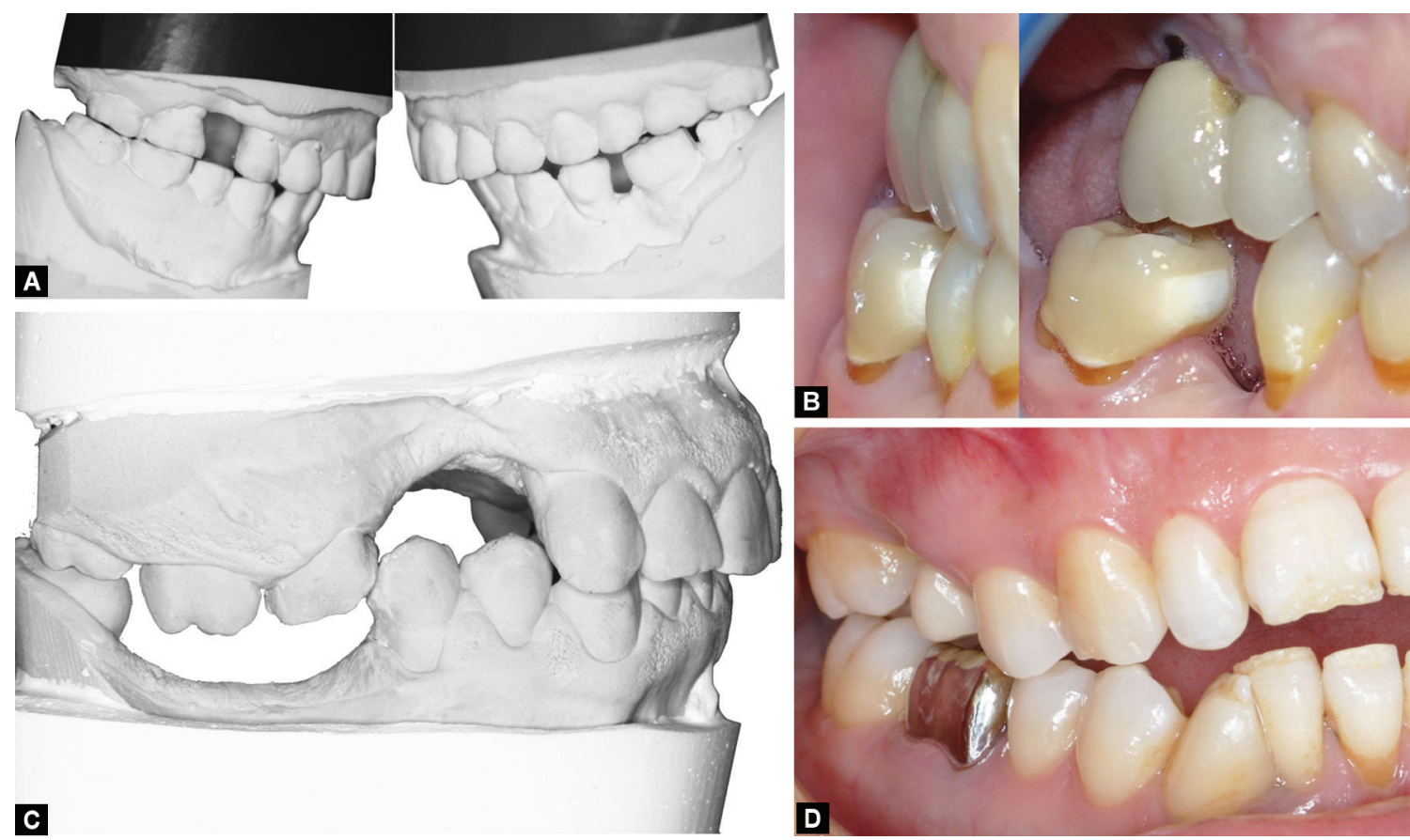

Figs 3A to D: Mandibular instability, with: (A) ICP: Global instability; (B) ICP: Infraocclusion: A new crown on 47 without contact; (C) ICP: Loss of posterior occlusal support; (D) ICP: Anterior occlusal instability

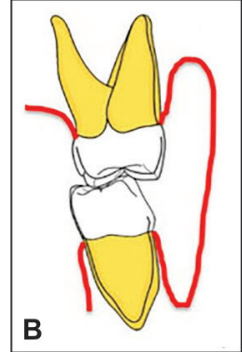

Normocclusion

High stability

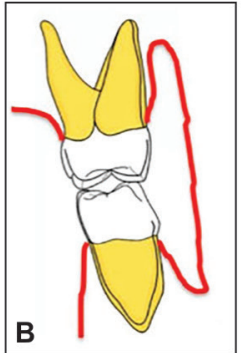

Edge-to-edge occlusion (half cusp)

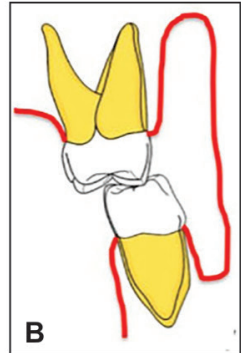

Reversed occlusion (one cusp)

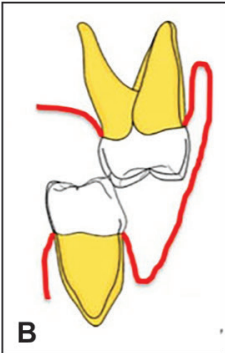

Scissor occlusion (two cusps)

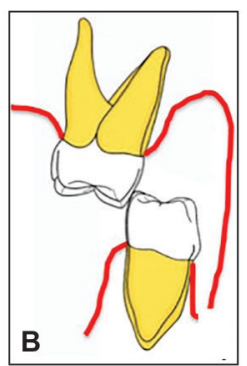

Reversed scissor occlusion (two cusps)

No stability

No stability

Fig. 4: Transverse posterior occlusal relationship in the ICP: Five diagnostic categories (frontal view of left molars-B, buccal)

without risk of "joint pinch," the muscles being used under optimal synergistic conditions. In the ICP, the mandibular position must be transversely "centered," which means "orthopedically stable," especially in the transverse direction. The centering function is more characterized by a transverse condylar centering than a sagittal centering of the condylar head in the mandibular fossa (Fig. 5A).

\section{Transverse Decentering}

The discrepancy (called delta) between the condylar positions in the CR (RCP) and the ICP is analyzed. A slight sagittal delta is very common in natural dentition and well-tolerated, but such a delta in the transverse direction seems riskier. Some authors note the predominance of signs of mechanical stress in the upper lateral third of the TMJ where the joint space and disc are always thinner. ${ }^{14-21}$ Electronic condylography performed on 4,528 subjects showed a sagittal RCP or ICP positional discrepancy 10 times greater than that observed transversely. ${ }^{22}$ Because this thin joint space is probably less tolerant, a lateral pinching is probably riskier than a sagittal condylar retroposition. A small transverse RCP or ICP positional discrepancy could constitute an orthopedic instability that generates a protective muscular response. Before studying this hypothesis, it must first be possible to measure the transverse delta; this is very rarely assessed in the studies classically cited to evaluate the relationship between TMD and malocclusion. ${ }^{23-27}$

Transverse mandibular decentering corresponds to an ICP that causes a lateral mandibular shift with respect to the condylar positions in CR, generating probably joint stresses (Fig. 5B). The centering analysis is static, comparing the mandibular position in the RCP and the ICP; decentering (deflected ICP) is detected clinically and then possibly confirmed by occlusal analysis on an articulator. Evaluation of the RCP-ICP delta is pointless in the face of an acute inflammatory musculo-articular condition. 


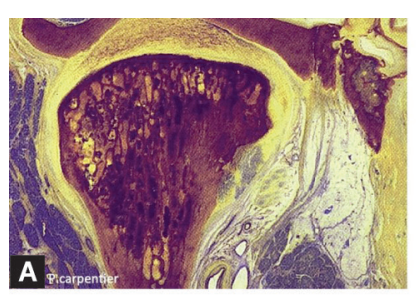

Articular stability (CR)

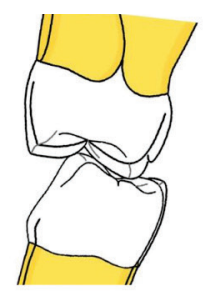

Maximal intercuspation occlusal stability

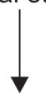

Mandibular stability

Muscle facilitation
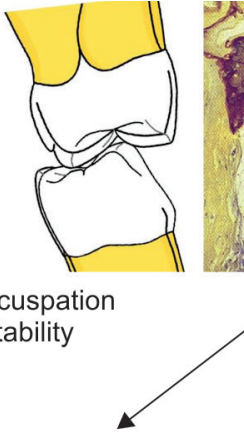

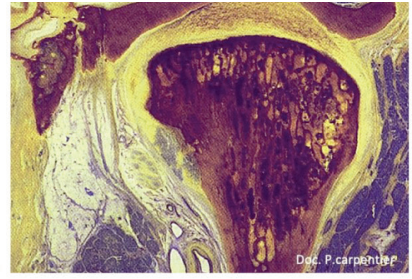

Articular stability (CR)
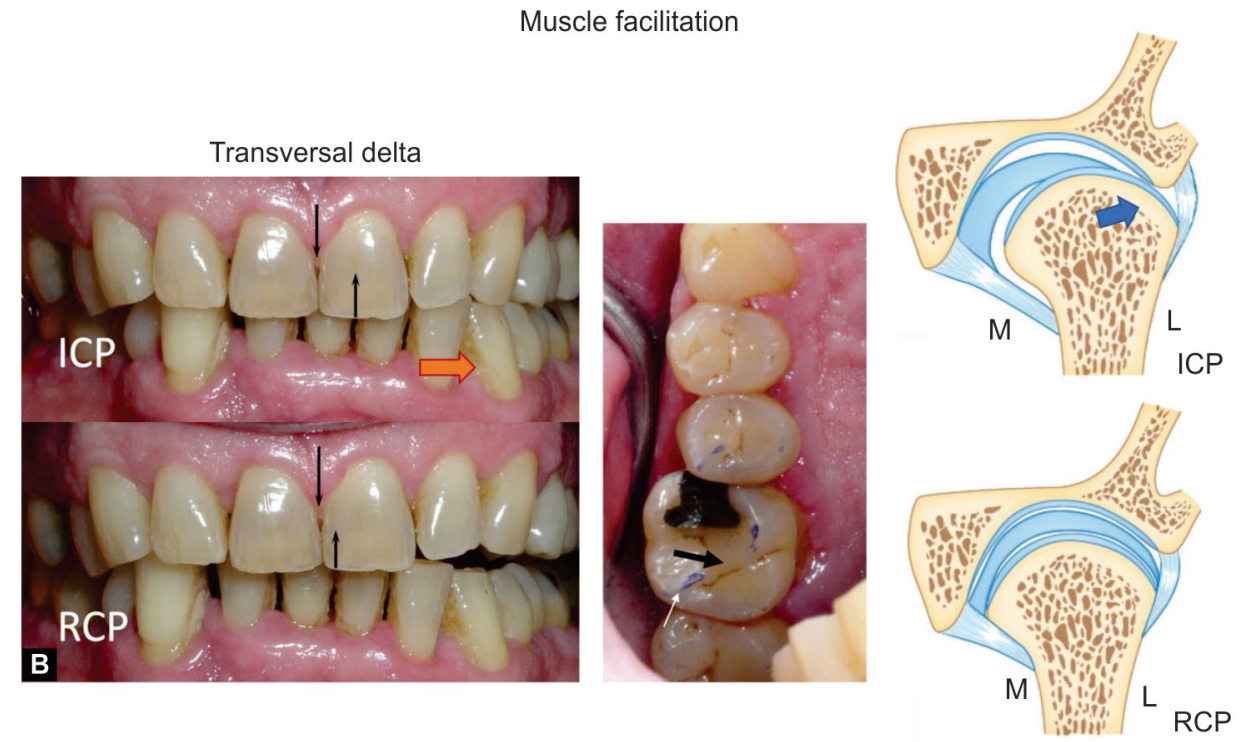

Figs 5A and B: Centering and decentering between the ICP and the RCP, with: (A) Transverse orthopedic concordance between the ICP and the TMJ; (B) Left transverse decentering: A transverse discrepancy between the RCP and the ICP could cause compression of the left lateral pole of the TMJ

\section{Dysfunctions of Occlusal Centering}

A sagittal RCP-ICP delta is physiological, most often of around $0.3 \mathrm{~mm}$ (at condylar level). The manducatory apparatus seems to tolerate a significantly larger sagittal delta quite easily. ${ }^{28}$ The physiological transverse delta is almost zero. ${ }^{29,30}$ Three centering dysfunctions can be identified keeping in mind that in the sagittal plane there are naturally large individual variabilities.

\section{Centering Dysfunctions}

- Transverse decentering: Transverse RCP-ICP delta.

- Anterior sagittal decentering: Excessive sagittal RCPICP delta (sagittal sliding $>2 \mathrm{~mm}$ ).

- Posterior sagittal decentering: Retroposition (excessively posterior condylar position).

\section{Guiding Functions or Dysfunctions}

\section{Guiding Function}

Mandibular reocclusion movements occurring in the eccentric mandibular position, that is, outside the $\mathrm{ICP}^{31}$ are directly influenced by occlusal guiding surfaces. When these contacts are predominantly anterior, they probably define an optimal occlusal guiding function (control) because of its efficiency (lower load)..$^{31,32}$ Like an access cone, the guiding surfaces control the mandibular movements toward one target position (ICP) avoiding eccentric posterior occlusal frictions and facilitating neuromuscular coordination.

\section{We distinguish the following guiding functions:}

- The sagittal guiding function: In protrusion or retrusion-on the proximal ridges of the maxillary incisors, and the distal crest of the maxillary canines;

- The lateral guiding function: Canine guide in the lateral excursion (laterality) - on the mesial part of the median ridge of the maxillary canine (in Angle's class I);

- Retrusive control: In retrusion or protrusion, the occlusal contacts along the RCP-ICP path are preferably located on the mesial part of the lingual cusp of the first maxillary premolar. This cusp displays a steep, overhanging slope, a true antiretrusive barrier. $^{33,34}$

\section{Occlusal Interference}

Some arguments advocate for a canine function, for example:

- Anterior guidance best distributes eccentric loads and generates the lowest level of stress concentration within the condyles when compared to group function guidance. ${ }^{32,35}$ 
- The laterotrusive position with canine guidance, in contrast to the group function, produces significantly lower elevator muscle activity. ${ }^{36}$

Although several studies of occlusal guidance in natural teeth have been published, we have not found sufficient evidence to conclusively support the superiority of canine function versus group function in terms of clinical outcomes. ${ }^{37}$ The key problem is not the discussion of the question of canine function versus group function, both can be functional, but to know how to diagnose guiding dysfunctions. There is much confusion in the literature regarding the term "occlusal interference." For example, for some authors, artificial occlusal interference is in fact the creation of one overocclusion, which is a stabilizing dysfunction, not a guiding dysfunction. $^{38,39}$

With regard to laterotrusion, authors often confuse a posterior occlusal contact occurring simultaneously with canine contact with a real posterior occlusal interference disengaging all the other teeth. ${ }^{39-42}$ This confusion is really astonishing considering that true posterior occlusal interference was initially described by Ramfiord in 1961, illustrated by Posselt in 1968, and defined with great pertinence by Ingervall in 1972, as the presence of a posterior tooth causing disocclusion of the anterior teeth in protrusion or laterotrusion movements. ${ }^{43-45}$ Moreover, Minagi has shown that, unlike interference, simultaneous posterior occlusal contact ("balanced occlusion") may be a protective factor for the TMJ. ${ }^{46,47}$

\section{Dysfunctions of Occlusal Guiding}

The different types of guiding dysfunctions are easily distinguished (Fig. 6).

Globally, an occlusal interference is defined as an occlusal obstacle that limits or deviates mandibular translational movements (laterotrusion or protrusion). It is appropriate to differentiate posterior and anterior occlusal interference and also occlusal prematurity:
- Posterior occlusal interference is a posterior occlusal contact that disengages all the anterior teeth (incisors and canines) in a mandibular translational movement. Posterior occlusal interference can occur in protrusion or laterotrusion (working or nonworking interference).

- Anterior occlusal interference is an anterior overguidance that locks translational capacities and causes excessive posterior disocclusion in laterotrusion.

The anterior teeth functions of capturing and cutting up foodstuffs, in addition to mastication and phonation, involve condylar translation along with the prominences.

This movement is more or less facilitated depending on the inclination of the occlusal guiding surfaces; an overly steep incisal slope (or canine slope) will be responsible for overguidance by obliterating the intracoronal angle. This locking could induce the following:

- Inhibition of protrusion or laterotrusion movements.

- Difficulty with an end-to-end bite.

- Friction between large surface areas and, therefore, wear on antagonist anterior teeth.

- Mandibular retrofunction, by an avoidance reaction that disrupts the neuromuscular system and that can stretch the TMJ ligament system (joint distension). ${ }^{48}$ Canine overguidance creates a risk of increased lateral play of the TMJ (Bennett's movement). ${ }^{49,50}$

- Excessive immediate posterior disocclusion.

- Occlusal prematurity does not affect mandibular translation but relates to the movement of mandibular elevation in rotation (terminal axial movement) that produces occlusal contact in the $\mathrm{CR}$.

- Most often involving a molar or malpositioned anterior tooth (e.g., a canine in reversed occlusion)

- Inducing transverse mandibular decentering to access the ICP. (Table 1)

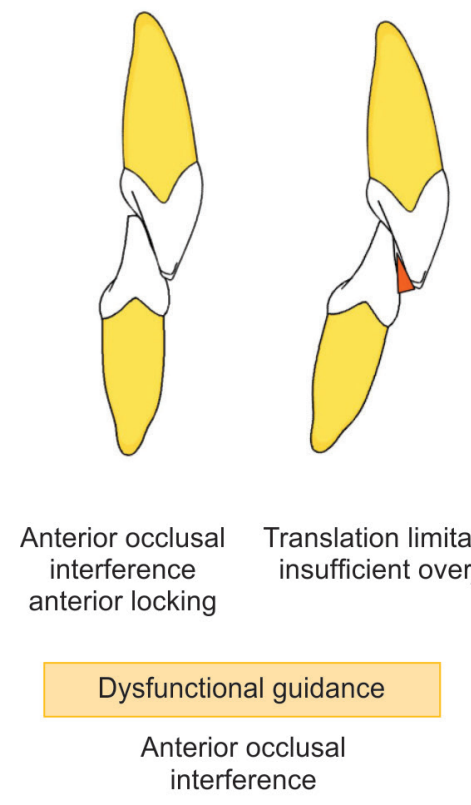

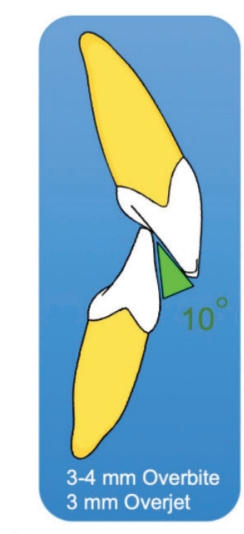

Freedom movements intracoronal angle $\left(10^{\circ}\right)$

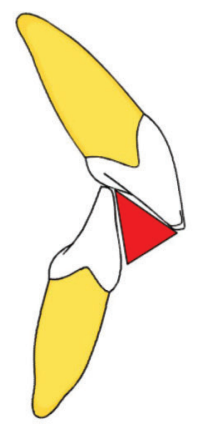

Excessive overjet insufficient guiding slope posterior occlusa interference

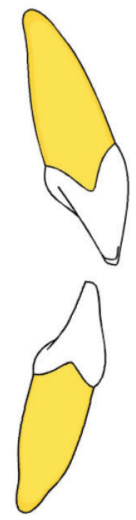

Insufficient overbite anterior open bite posterior occlusal interference

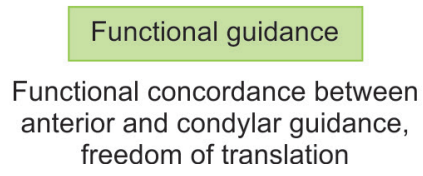

Fig. 6: Occlusal interference disengages the anterior teeth, while anterior occlusal interference restricts mandibular translational movements 
Table 1: Proposal for classification of occlusal functions, occlusal dysfunctions, and associated malocclusions

\begin{tabular}{|c|c|c|}
\hline \multirow{7}{*}{ Stabilizing function } & \multirow{7}{*}{ Unstable ICP } & Overocclusion \\
\hline & & Infraocclusion \\
\hline & & Dental arch instability \\
\hline & & Loss of posterior support \\
\hline & & Absence of anterior contact \\
\hline & & Excessive overjet or open bite \\
\hline & & Transverse occlusal disorders \\
\hline \multirow{3}{*}{ Centering function } & \multirow{3}{*}{ Deflected ICP } & Transversal RCP-ICP delta \\
\hline & & Excessive sagittal RCP-ICP discrepancy (posteroanterior sliding $>2 \mathrm{~mm}$ ) \\
\hline & & Retroposition (excessively posterior condylar position) \\
\hline \multirow{6}{*}{ Guiding function } & \multirow{6}{*}{ Occlusal interference } & Prematurity (manner of mandibular closure \\
\hline & & Posterior occlusal interference in protrusion \\
\hline & & Nonworking posterior occlusal interference in laterotrusion \\
\hline & & Working posterior occlusal interference in laterotrusion \\
\hline & & Anterior occlusal interference \\
\hline & & Full occlusal interference \\
\hline
\end{tabular}

\section{Discussion}

Analysis of occlusion only, without understanding the patient context, is not appropriate. Indeed, a claim to study the possible associations between symptomatology and malocclusion requires a detailed clarification of the different categories of occlusal dysfunctions that can be encountered. It is imperative that situations that may have very different pathogenic effects are not placed in the same category. Previously we noted some examples of significant nosological bias that discredits certain published research studies. This does not mean that the "mechanical" classification proposed above is sufficient to solve all the methodological problems of studies that deal with occlusion.

Evaluation of context is paramount; pathogenicity being most often the result of a combination of emotional tension, posttraumatic or systemic musculo-articular weakness, oral parafunctions, occlusal dysesthesia, and chronic pain.

Moreover, it seems important to clearly define a pathocclusion. Normocclusion is defined clearly in all textbooks. A pathogenic malocclusion could be an "occlusal relationship leading to deleterious dental or musculo-articular changes with degradation of the mandibular functions." It can be expressed via dental lesions (dental wear, fracture, abfraction, hypersensitivity, or bone lysis), occlusal dysesthesia, joint compression-distension, or muscle contractures. The boundary between eufunction and pathofunction is blurred, influenced by the patient's capacities for tolerance.

Furthermore, natural malocclusion should not be confused with iatrogenic malocclusion. Indeed, it is important to keep in mind that from the age of 6 years, installation of the natural occlusion of the adult subject in the permanent dentition requires almost 15 years. Malocclusions are very common, but they are established very gradually, during the very high potential for adaptation in young subjects. Mandibular growth and TMJ development occur simultaneously with the establishment of occlusal relationships. This occurs in the specific functional context of subjects with sometimes dysfunctional oral parafunctions. Despite the frequent presence of natural malocclusions, there are few symptomatic expressions of TMD during the growth period, and little thereafter. In young adult, mechanical stress increased by dysfunction-parafunctions may sometimes promote symptomatic expression due to the negative progression of a multifactorial combination (psychological, biological, and mechanical) that destroys the existing biomechanical balance. This is a natural development and is probably quite rare. In contrast, therapeutic interventions (extractions, restorations, prosthodontics, and orthodontics) impact the masticatory apparatus via sudden changes that require immediate adaptation, which can exceed the tolerance capacities of the system. Occlusal analysis must always be situated in its global historical context, so the two opposite occlusal contexts must not be confused:

- The progressive onset of occlusal disorders throughout life

- The sudden onset of traumatic or iatrogenic occlusal disorders.

In addition, healthy and deteriorated dental systems should not be confused. In a natural, healthy dental system, even with occlusal disorders, occlusal treatment for TMD is generally not recommended. But, in the case of reconstruction (only because of dental status), optimization of occlusal functions remains the rule even in the absence of concrete proof of a link with TMD. This is the simple application of the elementary precautionary principle. Occlusion is the key to optimizing the chewing and swallowing functions of the dental system and its stability. It influences diet, nutrition, swallowing as well as phonation, and esthetics. In addition, occlusion plays a role in cognition and well-being. Some authors report that there is no strong evidence to support a particular occlusal design and the establishment of definitive clinical guidelines. ${ }^{20,40}$ Common sense must be the order of the day when choosing clear occlusal criteria that are simple to implement and as close as possible to physiological principles.

If dental treatments are necessary, probably they must optimize occlusal functions to promote maintenance of the structures (TMJ, muscles, bones, and teeth) and to facilitate oral functions.

\section{Conclusion}

Occlusal functions are considerably more adaptable than is conventionally believed. The idea that a TMD would be triggered by a slight occlusal anomaly is not scientifically supported. But the topic remains confused, and a guideline is needed for the simple analysis of occlusal relationships and for when an occlusal reconstruction is indicated because of the dental 
status of the patient. The practitioner, in daily practice, needs occlusal references to orient treatment. A precise classification of occlusal functions or dysfunctions allows the diagnosis of occlusal abnormalities. This occlusal analysis method could then be used by a large number of practitioners, with a view to align the research of epidemiological studies focused on the role of occlusion, in order to obtain results in different studies that are capable of being compared.

\section{Clinical Significance}

Occlusal analysis using the three occlusal functions of "stabilizing, centering, guiding" can be of great help in avoiding incorrect interpretations. So, this occlusal function classification could be relevant to many fields, for instance, for epidemiological studies dealing with TMD and occlusion, or orthodontic treatment as well as in everyday practice.

\section{ACKnowledgments}

This work is an original research article. The manuscript has not been published and will not be submitted elsewhere for publication while under consideration by the journal. The images displayed are original and have not been taken from any website or textbook.

\section{References}

1. Manfredini D, Lombardo L, Siciliani G. Temporomandibular disorders and dental occlusion. A systematic review of association studies: end of an era? J Oral Rehabil 2017;44(11):908-923. DOI: 10.1111/joor.12531.

2. Turp JC, Greene CS, Strub JR. The dental occlusion: a critical reflection on past, present and future concepts. J Oral Rehab 2008;35:502-512. DOI: 10.1111/j.0305-182X.2007.01820.x.

3. Steenks MH. The gap between dental education and clinical treatment in temporomandibular disorders and orofacial pain. J Oral Rehabil 2007;34(7):475-477. DOI: 10.1111/j.1365-2842.2007.01711.x.

4. Christensen C. Occlusion confusion. Dentaltown 2006;7(4):8-14.

5. The glossary of prosthodontic terms: ninth edition. J Prosthet Dent 2017;117:e1-e105. DOI: 10.1016/j.prosdent.2016.12.001.

6. Forssell H, Kalso E. Application of principles of evidence-based Medicine to occlusal treatment for Temporomandibular disorders: are there lessons to be learned. J Orofac Pain 2004;18(1):9-22. PMID: 15022533.

7. Skármeta N. Occlusal stability and mandibular stability: the major part of dentistry we are still neglecting. Cranio 2017;35(4):201-203. DOI: 10.1080/08869634.2017.1329686.

8. de Kanter RJAM, Battistuzzi PGFCM, Truin GJ. Temporomandibular disorders: "Occlusion" Matters! Pain Res Manag 2018;2018:8746858. DOI: $10.1155 / 2018 / 8746858$.

9. Möller E, Sheikholeslam A, Lous I. Response of elevator activity during mastication to treatment of functional disorders. Scand J Dent Res 1984;92(1):64-83. DOI: 10.1111/j.1600-0722.1984.tb00861.x.

10. Ferrario VF, Serrao G, Dellavia C, et al. Relationship between the number of occlusal contacts and masticatory muscle activity in healthy young adults. Cranio J Craniomandib Pract 2002;20(2):91-98. DOI: 10.1080/08869634.2002.11746196.

11. Forrester SE, Allen SJ, Presswood RG, et al. Neuromuscular function in healthy occlusion. J Oral Rehabil 2010;37(9):663-669. DOI: 10.1111/j.1365-2842.2010.02097.x

12. Kaidonis JA, Ranjitkar S, Lekkas D, et al. Functional dental occlusion: an anthropological perspective and implications for practice. Aust Dent J 2014;59(Suppl. 1):162-173. DOI: 10.1111/adj.12133.

13. Kaifu Y, Kasai K, Towsend G, et al. Tooth wear and the "design" of the human dentition: A perspective from evolutionary medicine. Yearb Phys Anthropol 2003;46:47-61. DOI: 10.1002/ajpa.10329.
14. Michelotti A, lodice $G$, Piergentili $M$, et al. Incidence of temporomandibular joint clicking in adolescents with and without unilateral posterior cross-bite: a 10-year follow-up study. J Oral Rehabil 2016;43(1):16-22. DOI: 10.1111/joor.12337.

15. Okeson JP. Management of temporomandibular disorders and occlusion. 7th ed. St Louis: Elsevier Mosby; 2014. 575 p.

16. Oberg T, Carlsson G, Fajers C. A morphologie study on a human autopsy material. Acta Odontol Scand 1971;29(3):349-384. DOI: 10.3109/00016357109026526.

17. Hansson T. Temporomandibular joint changes related to dental occlusion. In: Temporomandibular joint problems biology, diagnosis and treatment. Quintessence Publishing Co. ed; 1980.

18. Bates RE, Gremillion HA, Stewart CM. Degenerative joint disease. Part I: diagnosis and management considerations. Cranio 1993;11(4):284290. DOI: 10.1080/08869634.1993.11677980.

19. Bates RE, Gremillion HA, Stewart CM. Degenerative joint disease. Part II: symptoms and examination findings. Cranio 1994;12(2):88-92. DOI: 10.1080/08869634.1994.11678000.

20. He SS, Deng X, Wamalwa P, et al. Correlation between centric relationmaximum intercuspation discrepancy and temporomandibular joint dysfunction. Acta Odontol Scand 2010;68(6):368-376. DOI: 10.3109/00016357.2010.517552.

21. Wang C, Yin X. Occlusal riskfactors associated with temporomandibular disorders in young adults with normal occlusions. Oral Surg Oral Med Oral Pathol Oral Radiol 2012;114(4):419-423. DOI: 10.1016/j. oooo.2011.10.039.

22. Slavicek R. The masticatory organ : functions and dysfunctions. Gamma edit. Klosterneuburg, Austria; 2002.

23. Türp JC, Schindler H. The dental occlusion as a suspected cause for TMDs: epidemiological and etiological considerations. J Oral Rehabil 2012;39(7):502-512. DOI: 10.1111/j.1365-2842.2012.02304.x.

24. Manfredini D, Stellini E, Gracco A, et al. Orthodontics is temporomandibular disorder-neutral. Angle Orthod 2015;86(4):649654. DOI: 10.2319/051015-318.1.

25. Pullinger AG, Seligman DA. Quantification and validation of predictive values of occlusal variables in temporomandibular disorders using a multifactorial analysis. J Prosthet Dent 2000;83(1):66-75. DOI: 10.1016/ s0022-3913(00)70090-4.

26. Sousa ST de, Mello VVC de, Magalhães BG, et al. The role of occlusal factors on the occurrence of temporomandibular disorders. Cranio 2015;33(3):211-216. DOI: 10.1179/2151090314Y.0000000015

27. Manfredini D, Perinetti G, Stellini E, et al. Prevalence of static and dynamic dental malocclusion features in subgroups of temporomandibular disorder patients: implications for the epidemiology of the TMD-occlusion association. Quintessence Int 2015;46(4):341-349. DOI: 10.3290/j.qi.a32986.

28. Rosner D, Goldberg GF. Condylar retruded contact position and intercuspal position in dentulous patients. Part II: patients classified by amnestic questionnaire. J Prosth Dent 1986;56(3):359-368. DOI: 10.1016/0022-3913(86)90020-x.

29. Suzuki K, Mito T, Ishizaki K, et al. Mandibular lateral translation during symmetric mandibular function in relation to patterns of intracapsular derangement of TMJ. J Stomat OccMed 2009;2(1):16-23. DOI: 10.1007/s12548-009-0003-2.

30. Mito T, Ishiazaki K, Suzuki K, et al. Mandibular lateral translation during symmetric mandibular movement. J Stomat OccMed 2009;2:24-31. DOI: 10.1007/s12548-009-0004-1.

31. Ogawa T, Ogawa M, Koyano K. Different responses of masticatory movements after alteration of occlusal guidance related to individual movement pattern. J Oral Rehab 2001;28(9):830-841. DOI: 10.1111/j.1365-2842.2001.00672.x.

32. Williamson EH, Lundquist DO. Anterior guidance: its effect on EMG activity od the temporal and masseter muscles. J Prosth Dent 1983;49(6):816-822. DOI: 10.1016/0022-3913(83)90356-6.

33. Gausch K, Kulmer S. The role of retro-disclusion in the treatment of the TMJ patient. J Oral Rehabil 1977;4(1):29-32. DOI: 10.1111/j.13652842.1977.tb00960.x. 
34. Gausch K, Kulmer S, Moser F, et al. Richtlinien der ARGE prothetik und gnathologie für eine zeitgemässe diagnostik des kau-sytems. Osterr Zahnärzte-Ztg 1979;(31):31:12-16.

35. Standlee JP, Caputo AA, Ralph JP. Stress transfer to mandible during anterior guidance and group function eccentric movements. J Prosth Dent 1979;41(1):35-39. DOI: 10.1016/0022-3913(79)90353-6.

36. Miralles R, Manns A, Nass X, et al. Influence of protrusive functions on electromyographic activity of elevator muscles. Cranio 1987;5(4):324332. DOI: 10.1080/08869634.1987.11678207.

37. Koyano K, Tsukiyama K, Kuwatsuru R. Rehabilitation of occlusion science or art? J Oral Rehabil 2012;39(7):513-521. DOI: 10.1111/j.13652842.2012.02303.x.

38. Clark GT, Tsukiyama Y, Baba K, et al. Sixty-eight years of experimental occlusal interference studies: what have we learned? J Prosthet Dent 1999;82(6):704-713. DOI: 10.1016/s0022-3913(99)70012-0.

39. Michelotti A, Farella M, Gallo L, et al. Effect of occlusal interferences on habitual activity of human masseter. J Dent Res 2005;84(7):644-648. DOI: $10.1177 / 154405910508400712$

40. Marklund S, Wänman A. A century of controversy regarding the benefit or detriment of occlusal contacts on the mediotrusive side. J Oral Rehabil 2000;27(7):553-562. DOI: 10.1046/j.13652842.2000.00629.x.

41. Seligman DA, Pullinger. Association of occlusal variables among refined TM patient diagnostic groups. J Craniomandib Disord 1989;3(4):227-236. PMID: 2639160.
42. Marklund S, Wänman A. A century of controversy regarding the benefit or detriment of occlusal contacts on the mediotrusive side. J Oral Rehab 2000;27(7):553-562. DOI: 10.1046/j.1365-2842.2000.00629.x.

43. Ramfjord S. Bruxism, a clinical and electromyographic study. J Am Dent Ass 1961;62:21-44. DOI: 10.14219/jada.archive.1961.0002.

44. Posselt U. Physiologie de l'occlusion et réhabilitation. Paris: J. Prélat; 1968. $358 \mathrm{p}$.

45. Ingervall $\mathrm{B}$. Tooth contacts on the functionnal and non functionnal side in children and youngs adults. Arch Oral Biol 1972;17:191-195. DOI: 10.1016/0003-9969(72)90147-1.

46. Minagi S, Watanabe H, Sato TTH. The relationship between balancingside occlusal contact patterns and TM joint sounds in humans. J Craniomandib Disord 1990;4(4):251-256. PMID: 2098402.

47. Minagi S, Ortsuki $\mathrm{H}$, Sato T, et al. Effects of balancing-side occlusion on the ipsilateral TMJ dynamics under clenching. J Oral Rehab 1997;24(1):57-61. DOI: 10.1046/j.1365-2842.1997.00431.x.

48. Isberg AM, Isacsson G. Tissue reactions of the temporomandibular joint following retrusive guidance of the mandible. Cranio 1986;4(2):143-148. DOI: 10.1080/08869634.1986.11678139.

49. Toubol JP, Michel JF.Lemouvement initial de Bennett: expérimentation clinique, conséquences thérapeutiques. Cah Proth 1983;(42):69-87. PMID: 6590102.

50. Ogawa M, Ogawa T, Koyano K, et al. Effect of altered canine guidance on condylar movement during laterotrusion. Int J Prosthodont 1998;11(2):139-144. PMID: 9709603. 